\title{
ANALISE DOS INSTRUMENTOS DE PLANEJAMENTO DE GESTÃO DE UM MUNICÍPIO DO SERTÃO DE PERNAMBUCO
}

\author{
T. L. de ALMEIDA ${ }^{1}$, A.C. ALVES ${ }^{1}$, C. E. MAMEDES $^{1}$, M. A. Q. N. MALAQUIAS ${ }^{1}$, M. \\ V. M \\ . MOURA ${ }^{1}$, R. S. LEANDRO ${ }^{1}$, S. C. de MENEZES ${ }^{1}$ \\ ${ }^{1}$ Escola de Governo de Saúde Publica de Pernambuco, Residência em Saúde Coletiva \\ E-mail para contato: tatiana_lial@hotmail.com
}

\begin{abstract}
RESUMO - O trabalho trás uma analise dos instrumentos de gestão de um município do sertão de Pernambuco. Os instrumentos avaliados são: o plano municipal de saúde, o plano anual de saúde e o relatório anual de saúde; é analisado o plano municipal de saúde do periodo 2014-2017 e os demais instrumentos do período de 2014 a 2016. Observou-se que existem diversos erros na construção dos instrumentos, e inconstâncias entre o proposto no plano anual de saúde e o que consta no relatório anual de gestão.
\end{abstract}

Palavras Chave: Sistema Único de Saúde; Programação de saúde; gerenciamento; governança.

\begin{abstract}
The work brings an analysis of the management tools of a municipality in the hinterland of Pernambuco. The instruments evaluated are: the municipal health plan, the annual health plan and the annual health report; the municipal health plan for the period 2014-2017 and the other instruments for the period from 2014 to 2016 were analyzed. It was observed that there are several errors in the construction of the instruments, and inconsistencies between that proposed in the annual health plan and what is stated in the annual.
\end{abstract}

Keywords: Health Unic System; Health programming; management; governance.

\section{INTRODUÇÃO}

Os instrumentos de planejamento do Sistema Único de Saúde - SUS são de extrema importância para sua gestão. O Plano de Saúde é um instrumento de planejamento que define a implementação de ações no âmbito da saúde de cada esfera de gestão para um período de quatro anos. É responsável por consolidar as políticas e compromissos de saúde, a partir de uma análise situacional, das necessidades de saúde da população e de suas peculiaridades, além de nortear a elaboração do orçamento e do planejamento do governo relacionado à saúde. Deve ser elaborado no primeiro ano da gestão em curso, com execução a partir do ano subsequente (BRASIL, 2016). 
De acordo com $\S 3^{\circ}$ do Art. $3^{\circ}$ da Portaria $n^{\circ} 3.125$ de 25 de setembro de 2013, o Plano de Saúde será elaborado a partir das necessidades de saúde da população, considerando a analise situacional contidas no mapa de saúde, dentre os seguintes temas: estrutura do sistema de saúde; redes de atenção de saúde; condições sociossanitárias; fluxos de acesso; recursos financeiros; gestão do trabalho e da educação na saúde; ciência, tecnologia, produção e inovação em saúde e gestão.

Considerando que o Plano Municipal de Saúde deve ser apresentado nas reuniões do Conselho Municipal se faz necessário que nesse espaço haja um esclarecimento com relação a este documento, incluindo suas metas, objetivos e indicadores, para que o público votante composto por trabalhadores, usuários e gestores, tenha de fato conhecimento e entendimento para questionar, criticar e acrescer de forma positiva e construtiva ao Plano.

Outro instrumento utilizado pela gestão é a Programação Anual de Saúde (PAS), esta é responsável por operacionalizar as intenções contidas no Plano de Saúde e anualizar os compromissos e metas assumidos, prevendo a alocação e execução dos recursos orçamentários. O documento deve detalhar as ações e serviços; as metas anuais; os indicadores e os recursos orçamentários no ano. Deve ser elaborada no ano em curso e executada no ano subsequente (BRASIL, 2016).

Para Estados e Municípios a PAS disporá da seguinte estrutura: (i) a definição das ações que naquele ano, garantirão o alcance dos objetivos e o cumprimento das metas do Plano de Saúde; (ii) identificação dos indicadores que serão utilizados para o monitoramento da PAS; (iii) a previsão da alocação dos recursos orçamentários necessários ao cumprimento da PAS. (BRASIL, 2013a)

Após planejar e operacionalizar as ações de saúde faz-se necessário que o gestor preste contas das atividades propostas pela PAS e executadas no ano anterior, esta prestação é realizada no chamado Relatório Anual de Gestão (RAG). Este instrumento apresenta, portanto, os resultados que foram alcançados com o cumprimento da PAS e de acordo com as diretrizes, objetivos e indicadores do PMS, podendo ainda guiar possíveis redirecionamentos necessários ao Plano e às Programações seguintes (BRASIL, 2016).

Sendo assim, o RAG deve contemplar: I - as diretrizes, objetivos e indicadores do Plano de Saúde; II - as metas da PAS previstas e executadas; III - a análise da execução orçamentária; e IV - as recomendações necessárias, incluindo eventuais redirecionamentos do Plano de Saúde. (BRASIL, 2013a):

Levando em consideração a funcionalidade de cada instrumento acima citado, utilizado pela gestão, buscou-se fazer uma avaliação dos mesmos focando em um município do sertão de Pernambuco, com cerca de 36.547 habitantes (IBGE, 2014), sede de uma Regional de Saúde, onde é desenvolvido as atividades de um programa de residência multiprofissional em saúde coletiva.

\section{MATERIAIS E MÉTODOS}

A presente pesquisa foi realizada através de revisão de literatura e analise documental. Os documentos analisados (PS, PAS, RAG) estão disponíveis online através do site SARGSUS. Foram analisados o Plano Municipal de Saúde 2014-2017, os Planos Anuais de Saúde de 2014, 2015, e 2016 e seus respectivos Relatórios Anuais de Gestão. 
Em primeiro momento foi realizado a revisão de literatura, através de artigos e manuais do Ministério da Saúde. No segundo momento foi feita a leitura dos instrumentos (PS, PAS, RAG). Em seguida foi feita a analise comparativa do Plano Municipal de Saúde com os Planos anuais de saúde, para verificar se as metas estavam distribuídas anualmente. E por ultimo a analise dos planos anuais de saúde para verificar se os indicadores distribuídos nos planos anuais de saúde tinham sido alcançados ou não.

\section{RESULTADOS E DISCUSSÃO}

Desta forma a avaliação realizada no Plano Municipal de Saúde (PMS) de Afogados da Ingazeira levou-nos a inferir que o mesmo não apresenta uma análise da situação de saúde, somente apresenta os dados e indicadores de saúde sem interpretá-los ou coloca-los em evidência ou prioridade diante da realidade local. Além do mais, não foi definido no PMS as diretrizes, objetivos, metas e indicadores, nem o processo de monitoramento e avaliação relativos ao quadriênio, o que impossibilitou comparar o Plano com as Programações Anuais de Saúde (PAS) com os Relatórios Anuais de Gestão (RAG) de cada ano.

O RAG do ano (2014) possui em seu escopo um detalhamento das metas e seus respectivos indicadores por extenso, relativo ao cumprimento ou não das propostas da PAS (2014), apresentando muitas das metas cumpridas, e naquelas não cumpridas a sua respectiva justificativa, o que facilitou a análise e compreensão dos instrumentos (Figura 1 e 2).

Figura 1. Estrutura da PAS 2014.

\begin{tabular}{|c|c|c|c|}
\hline \multicolumn{4}{|c|}{ INDICADOR: } \\
\hline \multicolumn{4}{|c|}{ COBERTURA POPULACIONAL ESTIMADA PELAS EQUIPES DE ATENÇÃO BÁSICA } \\
\hline META 2014 & AÇ̊̊O & INDICADOR & RESPONSÁVEL \\
\hline Regularizar 03 ESF junto a CIR/CIB/MS. & $\begin{array}{c}\text { Aprovar projeto junto a CIR e encaminhar } \\
\text { proposta para CIB e MS da implantaçăo } \\
\text { de } 03 \mathrm{ESF} \text {. }\end{array}$ & $\begin{array}{l}\text { Manter } 100 \% \text { de cobertura populacional } \\
\text { pelas equipes de Atençáo Básica }\end{array}$ & AB/COORDENAÇÃO NASF MUNICIPAL \\
\hline Implantar 01 Equipe do NASF & $\begin{array}{c}\text { Elaborar projeto e aprovar junto ao } \\
\text { CMS/CIR/ClB e MS. }\end{array}$ & $\begin{array}{l}\text { Garantir } 100 \% \text { de cobertura das açōes } \\
\text { do NASF na zona urbana e rural }\end{array}$ & AB/COORDENAÇÃO NASF MUNICIPAL \\
\hline
\end{tabular}

Figura 2. Estrutura do RAG 2014.

\section{PROGRAMAÇÃO ANUAL DE SAÚDE}

1- Diretriz:DIRETRIZ 1 - GARANTIA DO ACESSO DA POPULAÇÃO A SERVIÇOS DE QUALIDADE, COM EQUIDADE E EM TEMPO ADEQUADO AO ATENDIMENTO DAS NECESSIDADES DE SAÚDE, MEDIANTE APRIMORAMENTO DA POLITICA DE ATENÇÃO BÁSICA E DA ATENÇÃO ESPECIALIZADA.

1.1- Objetivo:UTILIZAR MECANISMOS QUE PROPICIEM A AMPLIAÇÃO DO ACESSO A ATENÇÃO BÁSICA

Metas: REGULARIZAR 03 ESF JUNTO A CIR/CIB/MS.

Indicadores: MANTER 100\% DE COBERTURA POPULACIONAL PELAS EQUIPES DE ATENÇÃO BÁSICA

2- Diretriz:DIRETRIZ 1 - GARANTIA DO ACESSO DA POPULAÇÃO A SERVIÇOS DE QUALIDADE, COM EQUIDADE E EM TEMPO

Identificou-se que diante de um total de 59 metas, 21 (36\%) foram alcançadas, ficando 17 (29\%) metas a serem revistas e/ou reprogramadas. Entretanto, percebeu-se que 
algumas metas presentes na Programação Anual de Saúde não aparecem no Relatório de Gestão, e vice e versa, o quantitativo dessas metas corresponde a $29 \%$ do total. Tal fator causou estranheza aos leitores e denotou certo grau de descuido na organização e construção do documento.

Ao analisar a PAS e RG dos anos 2015 e 2016 a organização dos documentos mostra-se diferenciada, pois eles apresentam as ações realizadas a partir dos seus indicadores, sem o devido detalhamento de cada meta, como apresentado no RAG 2014, impossibilitando a comparação entre os documentos PAS e RAG de 2015 e 2016 (Figuras 3).

Figura 3. Estrutura do RAG 2015.

\section{Programação Anual de Saúde e Pactuação da Saúde}

Diretriz 1 - Garantia do acesso da população a servicos de qualidade, com equidade e em tempo adequado ao atendimento das necessidades de saúde, mediante aprimoramento da política de atenção básica e da atenção especializada.

Objetivo 1.1 - Utilização de mecanismos que propiciem a ampliação do acesso da atenção básica.

\begin{tabular}{|c|c|c|c|c|}
\hline $\mathbf{N}^{\circ}$ & Indicador & Meta 2015 & Resultado & Unidade \\
\hline 1 & $\begin{array}{l}\text { COBERTURA POPULACIONAL ESTIMADA PELAS EQUIPES DE } \\
\text { ATENÇÃO BÁSICA. }\end{array}$ & 100,00 & 100,00 & $\%$ \\
\hline 2 & $\begin{array}{l}\text { PROPORÇÃO DE INTERNAÇÕES POR CONDIÇÕES SENSIVEIS } \\
\text { A ATENÇÃ̃O BÁSICA (ICSAB) }\end{array}$ & 28,00 & 29,50 & $\%$ \\
\hline
\end{tabular}

Muitas das metas quantitativas apresentadas no RAG (2015) não se relacionam com as metas propostas na PAS (2015). Ainda, algumas unidades de medida propostas para as metas definidas na PAS divergem em sua forma de um documento para outro (PAS e RAG), assim como, não possibilitam medir o cumprimento da meta (Figura 4, 5, 6 e 7$)$.

Figura 4. Meta do RAG 2015 não coincide com a meta do Indicador da PAS 2015.

Figura 5. Unidade de medida da meta apresentada no RAG 2015 diverge com a meta proposta na PAS 2015.

\begin{tabular}{|c|c|c|c|}
\hline \multicolumn{4}{|c|}{ INDICADOR: } \\
\hline \multicolumn{4}{|c|}{ NÚMERO DECASOS NOVOS DE SIFILIS CONGÉNITA BM MENORES DE UM ANO DEIDADE } \\
\hline META 2015 & AÇÃo & INDICADOR & RESPONSÁVH \\
\hline \multirow{2}{*}{$\begin{array}{l}\text { Reduzír em } 60 \% \text { a incidência de s'́filis } \\
\text { congênta }\end{array}$} & $\begin{array}{l}\text { Realizar testes rápidos para s'rìis nas } \\
\text { UBSS's do munićpio }\end{array}$ & \multirow{2}{*}{ \% incidència de sf́fils congènita } & \multirow{2}{*}{$\begin{array}{l}\text { AB/COORDENA ÇÃO DE SAÚDE DA MULHRR E } \\
\text { COORDENAÇAO DESAUDE DA CRIANÇA }\end{array}$} \\
\hline & $\begin{array}{l}\text { Capactação das equipes de } \mathrm{AB} \text { em } \\
\text { acorselhamento pré e pós teste da s'tilis }\end{array}$ & & \\
\hline
\end{tabular}


Figura 6. Meta com indicador inadequado.

\begin{tabular}{|c|c|c|c|}
\hline \multicolumn{4}{|c|}{ INDICADOR: } \\
\hline \multicolumn{4}{|c|}{ COBERTURA DOS CENTROS DEATENÇÃO PSICOSSOCIAL } \\
\hline META 2015 & AÇÅOO & INDICADOR & RESPONSÁVE \\
\hline $\begin{array}{c}\text { Implantar o CAPS III (Regional), CAPS } \\
\text { AD III (Regional) e CA PS i }\end{array}$ & $\begin{array}{c}\text { Implantar os Servccos CAPS II, CAPS e } \\
\text { CPAS i }\end{array}$ & $\begin{array}{l}\text { \% Cobertura de Serviços de Atenção } \\
\text { psicossocial }\end{array}$ & COORDENAÇȦO DESAÚDE MENTAU SES/SAS-MS \\
\hline
\end{tabular}

Figura 7. Ação não se relaciona com a meta.

\begin{tabular}{|c|c|c|c|}
\hline \multicolumn{4}{|c|}{ INDICADOR: } \\
\hline & NÚMEERO DEÓBITOS MATERNOS & ETERMINADO PERIODO ELOC & ENCIA \\
\hline META 2015 & AÇĀo & INDICADOR & RESPONSÄVH \\
\hline $\begin{array}{c}\text { Implantar o Centro de Saúde da mulher } \\
\text { e da Criança }\end{array}$ & $\begin{array}{l}\text { Garantia de aces so ao pré-natal na } \\
\text { Attençáo Básca e na rede especializada }\end{array}$ & $\begin{array}{c}\text { No de Serviços Especializados } \\
\text { mplantados }\end{array}$ & AB/COORDENAÇÃO DE SAÚDE DA MULHER \\
\hline
\end{tabular}

Um caso em particular não adequou o Indicador com a meta, demonstrando uma falta de conhecimento sobre o assunto. Como mostrado a seguir.

Figura 8. Indicador inadequado à meta.

\begin{tabular}{|c|c|c|c|}
\hline \multicolumn{4}{|c|}{ INDICADOR: } \\
\hline \multicolumn{4}{|c|}{ PROPORÇÅO DE AÇŐES DE EDUCAÇÄO PERM ANENTEIMPLBMENTADAS EOU REALIZADAS } \\
\hline META 2015 & AÇÅOO & INDICADOR & RESPONSÁVE \\
\hline $\begin{array}{l}\text { Realzar concurso público para } \\
\text { preenchimento de vagas da secretria } \\
\text { municipal de saúde }\end{array}$ & $\begin{array}{l}\text { Realizar levantamento de necessidides } \\
\text { e realizar processo lictatório para } \\
\text { habiltar empresa para organizaçäo do } \\
\text { certame. }\end{array}$ & $\begin{array}{l}\text { No de profissionais que ingress aram no } \\
\text { Sistema Unico Municipal com vínculo } \\
\text { protegido. }\end{array}$ & $\begin{array}{l}\text { Dretoria de Recursos Humanos e Gestäo de Pessoas/Dretorizs } \\
\qquad \text { da SMS }\end{array}$ \\
\hline
\end{tabular}

Analisando a PAS e o RAG do ano de 2016, as compreensões e observações mostraram-se semelhantes ao ano de 2015. Percebeu-se que nem todas as ações que foram programadas e principalmente as que foram executadas na Programação Anual, foram abordadas no RAG de 2016, bem como ações que foram citadas no RAG não fazem parte do escopo das ações programadas na PAS. Observou-se também que não foi previsto a alocação dos recursos orçamentários necessários ao cumprimento das ações dentro da PAS.

Na Programação de 2016 foram encontradas 58 metas, entretanto o RAG do mesmo ano apresenta apenas 29 metas (50\%) e entre elas, cinco sem resultados; as demais metas presentes na PAS ficaram sem justificativa na RAG.

Figura 9. Indicador sem resultado.

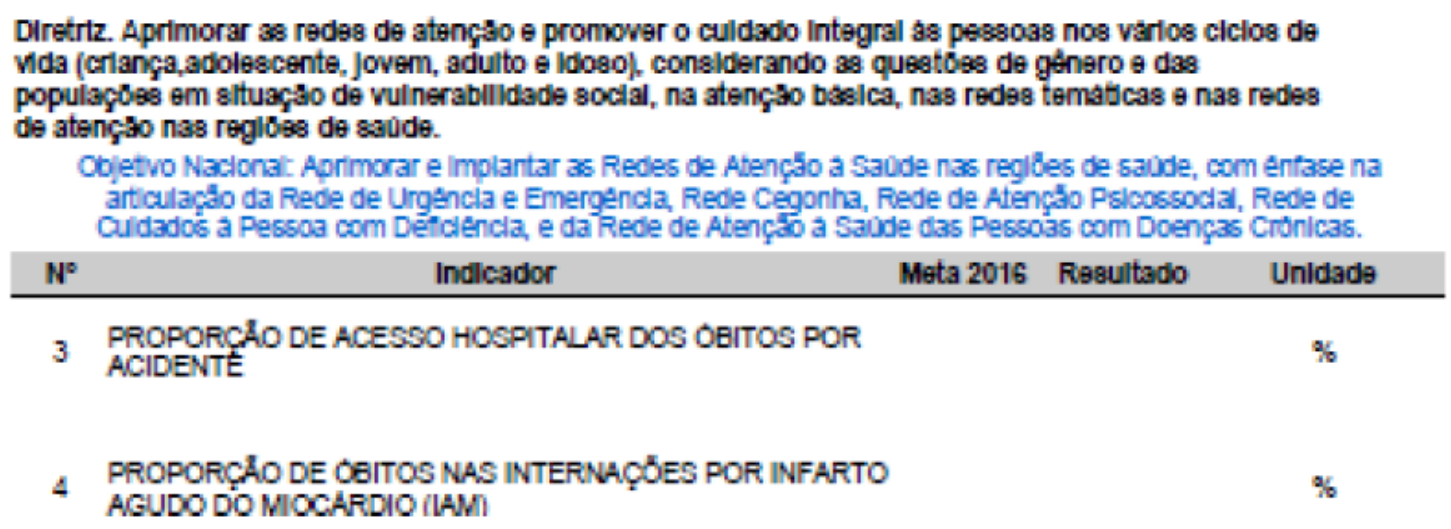


Diante da análise dos documentos percebeu-se a incompreensão do que seja um indicador, pois, em muitos casos ele não se relaciona com as metas e/ou não consegue medi-las. O conteúdo dos documentos também aparece de maneira incoerente em muitos casos, com divergências entre indicadores, metas e resultados, como foi visto anteriormente. Ressalta-se a grande diferença dos documentos entre os anos de 2014 e 2015.

Observa-se como relevante propor melhorias que contribuíssem para o a melhor elaboração dos documentos e consequentemente execução das ações propostas. Pode-se orientar como necessário a realização de reuniões entre as equipes para estudo e elaboração das Programações Anuais de Saúde e Plano Municipal de Saúde. Assim como a escolha de um profissional específico que fique responsável pela compilação dos dados obtidos nas reuniões. Nesses momentos deverá acontecer: (a) Inicialmente um estudo a respeito da forma de elaboração adequada dos documentos, contemplando todos os profissionais, incluindo os novos profissionais do quadro; (b) Integração das equipes para elaboração do Plano e Relatório e avaliação deste estudo do território e população; (c) Análise dos documentos anteriores com um olhar para o que já foi alcançado e as necessidades de saúde da população; (d) Melhor detalhamento das metas alcançadas e das que não foram alcançadas apresentando estas com justificativas que facilitem a analise posterior; (e) Estudo das unidades de medidas apropriadas para o alcance de cada meta; (f) Estudar estratégias de mobilização para participação ativa da população e dos profissionais na construção dos documentos; (g) Análise criteriosa dos documentos no Conselho Municipal de Saúde.

\section{CONCLUSÃO}

Diante do trabalho acima exposto, observaram-se entraves para a elaboração dos documentos: PMS, PAS, RAG. A utilização de referências importantes como a Portaria n³125/2013 foi precária, comprometendo assim construção de um documento coerente nos quesitos: análise situacional orientada, alocação dos recursos financeiros, definição das diretrizes, objetivos, metas e indicadores. Outro fator relevante foi a necessidade de uma análise fidedigna da situação de saúde local.

Percebeu-se também algumas falhas na elaboração dos documentos foram típicas da falta do olhar integrado entre as equipes, como verificado, por exemplo na inconsistência entre metas e indicadores entre os Planos e os Relatórios Anuais de Gestão.

Desta forma, considera-se a necessidade do fortalecimento de ações calcadas na pratica do planejamento, envolvendo todas as ações inclusive a elaboração dos documentos que nortearão o trabalho na rede de saúde. Acredita-se que atitudes remediativas serão cada vez mais extinguidas, sem falar que a alocação dos recursos será melhor direcionada, sem a presença de subfinanciamentos, beneficiando o trabalho dos profissionais e a assistência ofertada à população.

\section{REFERÊNCIAS}

AFOGADOS DA INGAZEIRA, Plano Municipal de Saúde, Secretaria Municipal de Saúde, 2014. 
AFOGADOS DA INGAZEIRA, Programação Anual de Saúde, Secretaria Municipal de Saúde, 2014.

AFOGADOS DA INGAZEIRA, Programação Anual de Saúde, Secretaria Municipal de Saúde, 2015.

AFOGADOS DA INGAZEIRA, Programação Anual de Saúde, Secretaria Municipal de Saúde, 2016.

AFOGADOS DA INGAZEIRA, Relatório Anual de Gestão, Secretaria Municipal de Saúde, 2014.

AFOGADOS DA INGAZEIRA, Relatório Anual de Gestão, Secretaria Municipal de Saúde, 2015.

AFOGADOS DA INGAZEIRA, Relatório Anual de Gestão, Secretaria Municipal de Saúde, 2016.

BRASIL. Manual de planejamento no SUS. Ministério da Saúde, Fundação Oswaldo Cruz. 1. ed., rev. - Brasília, 2016.

BRASIL. Portaria $\mathrm{n}^{\circ} 2.135$, de 25 de setembro de 2013. Estabelece diretrizes para o processo de planejamento no âmbito do Sistema Único de Saúde (SUS). Diário Oficial da União, Brasília, n. 187, 26 set. 2013a. Seção I. p. 60. Disponível em:< http://bvsms.saude.gov.br/bvs/saudelegis/gm/2013/prt2135_25_09_2013.html>.

VIEIRA, F. S. Avanços e desafios do planejamento no Sistema Único de Saúde. Ciência \& Saúde Coletiva, 14(Supl. 1):1565-1577, 2009. Disponível em: $<$ http://www.scielo.br/scielo.php?pid=S1413-

$81232009000800030 \&$ script $=$ sci_abstract\&tlng=pt $>$. 